\title{
COCYCLE FORMULAS FOR HOMOTOPY CLASSIFICATION; MAPS INTO PRO JECTIVE AND LENS SPACES $\left({ }^{1}\right)$
}

\author{
BY \\ PAUL OLUM
}

Introduction. In a previous work [8] we have introduced certain "secondlevel" invariants which bear on the homotopy classification and extension problems and, in particular, provide the machinery for an effective solution of these problems for the case of the second obstruction. It is our purpose here to show how these invariants may be explicitly computed in terms of cocycle formulas, simplicial or singular, provided that a cocycle formula is known for the relevant cohomology operation which enters. We apply these formulas then to give complete homotopy classification theorems for mappings of complexes (or manifolds) into real and complex projective spaces and into lens spaces.

By a homotopy classification theorem for the mappings of $X$ into $Y$ is meant a theorem which provides an effective procedure for determining whether two arbitrarily given maps $f, g: X \rightarrow Y$ are homotopic or not. Although many results are known which yield an "enumeration" of homotopy classes, the literature of topology contains extremely few such classification theorems $\left({ }^{2}\right)$ and our results will add somewhat to this list.

Since these results for mappings into projective and lens spaces are perhaps of most general interest here, we shall give them first in Part I below, deferring all proofs to Part II. Enumeration theorems for these mappings have been given before, but the homotopy classification has been known only in certain fortuitous special cases; references will be found at the beginning of each of the sections of Part I.

In the statement of the theorems of Part I we use rather extensively the notion of the difference homomorphism $(f-g)^{*}$ of two mappings $f$ and $g$. A detailed discussion of these difference homomorphisms will be found in [8, $\S 9]$. For convenient reference here we give in a brief appendix, $\$ 9$, a summary of their definition adequate for the present paper.

Part II will contain the general results on cocycle formulas for our second level invariants and, from these, proofs of the theorems of Part I. For Part II we shall assume that the reader is familiar with [8].

Throughout the paper all cochains, cocycles, etc. of a space $X$ are taken

Received by the editors June 13, 1961.

(1) This work was sponsored by the Office of Naval Research under Contract No. Nonr 401(20)-NR 043-167.

(2) For a general discussion of the enumeration and classification problems, see the introduction to [8]. 
in the singular complex $S(X)$, unless otherwise stated, and are assumed to be normalized, i.e. to vanish on degenerate simplices. If $z$ is a cocycle we use $\{z\}$ to denote its cohomology class.

When we consider a triangulated space we shall always suppose the vertices canonically ordered so that we may regard the simplicial complex as a subcomplex of the singular complex. Simplicial maps then will be taken to be order-preserving; this can always be achieved without altering the map by passing to the first barycentric subdivision.

Given a map $f: X \rightarrow Y$ we shall systematically use $f^{\prime \prime}$ for the induced cochain homomorphism (singular or simplicial) and $f^{*}$ for cohomology. There is always a chosen base point $x_{0}$ in the domain space, $y_{0}$ in the image space, and all maps carry $x_{0}$ into $y_{0}$. We use the notation " $f \cong g$ rel. $\omega$," for $\omega \in \pi_{1}\left(Y, y_{0}\right)$, to mean " $f$ is homotopic to $g$ with the image of $x_{0}$ moving through a loop representing $\omega$." For a subspace $A \subset X$, " $f \cong g$ rel. $A$ " has the usual meaning.

The product operation for cochains will be designated by the symbol "." and, to avoid ambiguity with the superscript notation for dimension, we shall always use a parenthesis in taking powers, e.g. $\left(z^{q}\right)^{k}$ for the $k$ th power of $z^{q}$.

\section{MAPS into PROJECTIVE AND LENS SPACES}

1. Maps of $q$-manifolds into real projective $q$-space. Let $M$ be a compact connected $q$-manifold and $P$ real projective $q$-space, with both spaces assumed triangulated. Let $A \subset M$ be a subcomplex containing the base point $x_{0}$.

We shall give here a homotopy classification theorem $\left({ }^{3}\right)$ for maps of $M$ into $P$. An enumeration of these homotopy classes was given earlier by the author in [7, §9] and also by Gordon [5], except that Gordon left one case open (see [7, footnote 10, p. 469]). However, the homotopy classification was given in [7] only for the case of orientation-true maps $\left({ }^{4}\right)$; for non-orientationtrue maps some partial results were achieved using the group-ring degree $[7$, $\$ 18]$ but these were very limited.

For maps of an arbitrary $q$-complex (not necessarily a manifold) into $P$, see (3.2e) below.

Let $y^{1}$ generate $H^{1}\left(P ; Z_{2}\right)$, with $z$ a representative simplicial cocycle, and let $y^{q}$ generate the infinite cyclic group $H^{q}\left(P ; Z^{t}\right)$, where $Z^{t}$ denotes twisted integer coefficients $[7, \S 3]$; see also $\S .9$ below. Let $f$ and $g$ be simplicial maps: $M \rightarrow P$ with $f|A=g| A$.

(3) A part of this problem (the case where $q$ is odd) was considered by Wada in [11]; however, as pointed out by Wada himself in [12], the main extension and classification theorems of [11] are unfortunately not correct.

( ${ }^{4}$ ) In a connected manifold $M$, let $\pi_{1}^{0}\left(M, x_{0}\right)$ denote the subgroup of $\pi_{1}\left(M, x_{0}\right)$ consisting of "orientation-preserving" elements. This is all of $\pi_{1}\left(M, x_{0}\right)$ if $M$ is orientable; otherwise it is the image under projection of the fundamental group of the orientable 2-fold covering of $M$. We call $f: M \rightarrow M^{\prime}$ orientation-true provided that, for all $\gamma \in \pi_{1}\left(M, x_{0}\right), f_{*}(\gamma)$ is in $\pi_{1}^{0}\left(M^{\prime}, y_{0}\right)$ if and only if $\gamma$ is in $\pi_{1}^{0}\left(M, x_{0}\right)$. 
THEOREM 1.1. $f \cong g$ rel. $A$ if and only if

(i) $(f-g) * y^{1}=0$. see $\$ 9$.

(ii) $(f-g) * y^{q}=0$ where $(f-g) *$ is taken in the sense of local coefficients;

(iii) The element of $H^{q}\left(M, A ; Z_{2}\right)$ represented by the cocycle

$$
\sum_{k=0}^{q}(f+z)^{k} \cdot c \cdot(g+z)^{q-k}
$$

is zero, where $c \in C^{0}\left(M, A ; Z_{2}\right)$ is the (unique) simplicial cochain such that $\delta c=f^{\sharp} z-g^{\prime} z$.

THEOREM 1.2. $f \cong g$ rel. $\omega$, for $\omega \neq 0$ in $\pi_{1}(P)$, if and only if

(i) $f^{*} y^{1}=g^{*} y^{1}$,

(ii) $f^{*} y^{q}=(-1)^{q+1} g^{*} y^{q}$ (local coefficients),

(iii) the element of $H^{q}\left(M, x_{0} ; Z_{2}\right)$ from (iii) of Theorem 1.1 , with $A=x_{0}$, is equal to $(q+1) f^{*}\left(y^{1}\right)^{q}$.

(1.3) AdDenda AND Remarks: (a) Arbitrary continuous maps. If $f$ and $g$ are just continuous (not simplicial) maps above, then we take $z$ and $c$ as singular cochains; also there will be a singular cochain $v \in C^{q}\left(P ; Z_{2}\right)$ such that $(z)^{q+1}=\delta v$. Then Theorems 1.1 and 1.2 hold as stated except that the term $f^{t} v-g^{t} v$ must be added on to the expression in (iii).

(b) The possible difference homomorphisms. Suppose $(f-g)^{*} y^{1}=0$. Then $(f-g)^{*} y^{q}$ in $H^{q}\left(M, A ; Z^{\prime}\right)$ is divisible by 2 , where $Z^{f}$ denotes the local coefficient system in $M$ induced by $f$ from $Z^{t}$ in $P$; see $\$ 9$ for definition. Moreover, all elements of $H^{q}\left(M, A ; Z^{f}\right)$ divisible by 2 are realized for suitable $g$, with $f$ held fixed.

(c) Orientation-true maps. If $f$ and $g$ are orientation-true( $\left.{ }^{4}\right)$, then in Theorems 1.1 and 1.2 condition (iii) is superfluous and should be omitted. (Note, in particular, that this with (b) gives at once also the enumeration of homotopy classes in the orientation-true case.)

(d) Non-orientation-true maps. If $f$ and $g$ are not orientation-true, then condition (ii) in Theorem 1.2 is a consequence of condition (i) and may be omitted; (the same is true in Theorem 1.1 when $A=x_{0}$ ).

For non-orientation-true $f$ and $g$, the mappings $g$ such that $(f-g)^{*} y^{1}=0$ fall into distinct homotopy classes rel. $A$ which are in 1-1 correspondence with the elements( $\left.{ }^{5}\right)$ of $H^{q}\left(M, A ; Z^{f}\right)$. In particular, then, if $A=x_{0}$ there are two such classes. For odd $q$ these two classes remain distinct under free homotopy; for even $q$ they remain distinct under free homotopy if the mod 2 degree of $f$ is 0 and coincide if it is not 0 .

(5) It is not difficult to show that the mappings $g$ which satisfy both $(f-g)^{*} y^{1}=0$ and $(f-g)^{*} y^{q}=0$ fall into homotopy classes rel. $A$ in 1-1 correspondence with the elements of order 2 in $H^{q}\left(M, A ; Z^{f}\right)$. 
2. Maps into complex projective space. Let $X$ be a connected locallyfinite polyhedron of dimension $2 q+1$ and $\mathfrak{B}$ complex projective $q$-space (real dimension $2 q$ ), with both spaces assumed triangulated. Let $A \subset X$ be a subcomplex.

We give here the homotopy classification for maps of $X$ into $\mathfrak{B}$. The enumeration of these homotopy classes (which is a corollary of our resultssee (2.2b) below) was given by Wada in $[10]\left(^{6}\right)$; it follows also from Eckmann's work [2].

Let $y^{2}$ generate $H^{2}(\mathfrak{B} ; Z)$ and let $z$ be a representative simplicial cocycle. Let $f, g: X \rightarrow \mathfrak{B}$ be simplicial maps with $f|A=g| A$.

Theorem 2.1. $f \cong g$ rel. $A$ if and only if

(i) $(f-g) * y^{2}=0$,

(ii) the set of elements of $H^{2 q+1}(X, A ; Z)$ represented by the cocycles

$$
\sum_{k=0}^{q}\left(f^{\sharp} z\right)^{k} \cdot c \cdot\left(g^{\ddagger} z\right)^{q-k}
$$

for all $c \in C^{1}(X, A ; Z)$ such that $\delta c=f^{\sharp} z-g^{\sharp} z$ includes the element 0 .

(2.2) Addenda and Remarks. (a) Continuous maps. For $f, g$ just continuous, take $z$ and $c$ as singular and add the term $-\left(f^{\sharp} v-g^{\ddagger} v\right)$ to the expression in (ii) where $(z)^{q+1}=\delta v$.

(b) Enumeration. Let $\Lambda_{f, f}$ be the subgroup of $H^{2 q+1}(X, A ; Z)$ given by

$$
(q+1) f^{*}\left(y^{2}\right)^{q} \cdot H^{1}(X, A ; Z) .
$$

Then the maps $g$ such that $(f-g)^{*} y^{2}=0$ fall into distinct homotopy classes rel. $A$ which are in $1-1$ correspondence with $H^{2 q+1}(X, A ; Z) / \Lambda_{f, f}$.

(c) Restatement of theorem. The elements of (ii) above form a coset of the subgroup $\Lambda_{f, f}$ of (b). Hence, if $d(f, g)$ is a particular element given by (ii) for one choice of $c$, then $f \cong g$ rel. $A$ if and only if $d(f, g)$ is in $\Lambda_{f, f}$.

3. Maps into lens spaces. Let $(X, A)$ be as in $\S 2$ and let $L$ be a triangulated $(2 q+1)$-dimensional lens space with fundamental group cyclic of order $l$; see $[7, \S 8]$. We shall give the homotopy classification for maps of $X$ into $L$. This was done before only for the case where $X$ was an orientable $(2 q+1)$-manifold; see $[7, \S \S 5,8]$ and $(3.2 \mathrm{c})$ below.

Let $y^{1}$ generate $H^{1}\left(L ; Z_{l}\right)$, with $z$ a representative simplicial cocycle, and let $y^{2 q+1}$ generate $H^{2 q+1}(L ; Z)$. Let $f, g: X \rightarrow L$ be simplicial maps with $f \mid A$ $=g \mid A$. Let $\eta: Z_{l} \rightarrow Z$ denote the transformation which assigns to each integer mod $l$ the corresponding integer $m$ with $0 \leqq m<l$; we denote by $\eta$ also the induced transformation: $C^{*}\left(X ; Z_{l}\right) \rightarrow C^{*}(X ; Z)$.

${ }^{(6)}$ There is an error in Wada's results in [10] due to a mistake in computation; his argument here however is basically correct. In his Theorem 1, p. $71,2^{k} C_{n+1, k}$ ought to be $C_{n+1, k}$; in Theorem 2, p. $74,2^{n}$ ought to be $n+1$. 
THEOREM 3.1. $f \cong g$ rel. $A$ if and only if

(i) $(f-g)^{*} y^{1}=0$,

(ii) $(f-g)^{*} y^{2 q+1}=0$,

(iii) the element of $H^{2 q+1}(X, A ; Z)$ represented by the cocycle

$$
\frac{1}{l^{q+1}} \sum_{k=0}^{q}\left(\delta \eta f^{t z}\right)^{k} \cdot\left(\eta f+z-\eta g^{*} z-\delta \eta c\right) \cdot\left(\delta \eta g^{f z}\right)^{q-k}
$$

is zero, where $c \in C^{0}\left(X, A ; Z_{l}\right)$ is the (unique) simplicial cochain such that $\delta c=f^{\prime} z-g^{f} z$.

Furthermore, for any $\omega \in \pi_{1}\left(L, y_{0}\right), f \cong g$ rel. $\omega$ if and only if $f \cong g$ rel. $x_{0}$.

(3.2) Addenda and Remarks. (a) Continuous maps. For $f, g$ just continuous, take $z$ and $c$ as singular and add the term $-\left(f^{t} v-g^{t} v\right)$ to (iii) where $(\delta \eta z)^{q+1}=l^{q+1} \delta v$.

(b) The possible difference homomorphisms. Suppose $(f-g)^{*} y^{1}=0$. Then $(f-g)^{*} y^{2 q+1}$ in $H^{2 q+1}(X, A ; Z)$ is divisible by $l$; moreover, all elements of $H^{2 q+1}(X, A ; Z)$ divisible by $l$ are realized for suitable $g$, with $f$ held fixed.

(c) Orientable manifolds, etc. The element in (iii) above is annihilated by multiplication by $l$. Hence, if $H^{2 q+1}(X, A ; Z)$ has no nonzero element whose order divides $l$ (e.g., if $X$ is an orientable manifold, or if $X$ is a nonorientable manifold and $l$ is odd), then condition (iii) is superfluous and may be omitted.

(d) Enumeration. The maps $g$ such that $(f-g)^{*} y^{1}=0$ fall into distinct homotopy classes rel. $A$ which are in 1-1 correspondence $\left({ }^{7}\right)$ with the elements of $H^{2 q+1}(X, A ; Z)$.

(e) Maps of complexes into real projective space. The case of maps: $(X, A)$ $\rightarrow P$, where $X$ is a $q$-complex and $P$ is real projective $q$-space, is included above for $q$ odd since $P$ is then a lens space. For even $q$ this is not true, and also the convenient passage to mod 2 coefficients which makes the results in $\$ 1$ for manifolds so simple is (as will be clear from the proofs in \$8) not available for general complexes.

It is, however, still possible to give a cochain formula for even $q$, but now local coefficients enter rather delicately and we shall simply sketch the result which corresponds to Theorem 1.1 without proof. Using the notation of Theorem 1.1, but replacing the manifold $M$ by a $q$-complex $X$, it turns out that $f \cong g$ rel. $A$ if and only if

(i) $(f-g)^{*} y^{1}=0$,

(ii) $(f-g)^{*} y^{q}=0$ (local coefficients),

(iii) the element of $H^{q}\left(X, A ; Z^{\prime}\right)$ represented by the cocycle

$$
-\sum_{k=0}^{q}(-1)^{k}(f z z)^{k} \cdot c \cdot\left(g^{f} z\right)^{q-k}
$$

(7) It is not difficult to show that the maps $g$ which satisfy both $(f-g)^{*} y^{1}=0$ and $(f-g)^{*} y^{2 q+1}$ $=0$ fall into homotopy classes rel. $A$ in $1-1$ correspondence with the subgroup of $H^{2 q+1}(X, A ; Z)$ annihilated by multiplication by $l$. 
is zero, where $c \in C^{0}\left(X, A ; Z_{2}\right)$ is the (unique) simplicial cochain such that $\delta c=f^{\sharp} z-g^{\sharp} z$. Here $f^{\sharp} z, g^{\sharp} z$ and $c$ are taken, as before, with mod 2 coefficients, but in forming (3.3) these coefficients are replaced by the corresponding integers 0 or 1 and the cochain product and sum in (3.3) are then determined in the usual way for integers; the resulting $q$-cocycle is taken, however, as a cocycle with local integer coefficients, the local system being precisely that determined by $f^{\sharp} z$ (i.e. the operation along a 1-simplex $\sigma_{1}$ is trivial or nontrivial according to whether $\left(f^{\sharp} z\right)\left(\sigma_{1}\right)=0$ or 1$)$. This cocycle (3.4) determines then an element of $H^{q}\left(X, A ; Z^{f}\right)$, where $Z^{f}$ is as in (1.3b), and it is this element which we use above.

\section{COCYCLE FORMULAS FOR SECOND LEVEL INVARIANTS}

4. Cocycle formulas. We consider a cohomology operation $w \in H^{q+1}(\Pi, n ; G)$; see $[1$, exposé 14$]$. For any semi-simplicial pair $(K, L)$ and element $h \in H^{n}(K, L ; \Pi)$ we denote the result of this operation by $w \vdash h$ in $H^{q+1}(K, L ; G)$.

DEFINITION 4.1. By a cocycle formula for $w$ we mean a transformation carrying normalized cocycles into normalized cocycles:

$$
C_{w}: Z^{n}(K, L ; \Pi) \rightarrow Z^{q+1}(K, L ; G),
$$

defined for all s.s. pairs $(K, L)$, such that

(a) $\left\{C_{w}(z)\right\}=w \vdash\{z\}$.

(b) For every s.s. map $\phi:\left(K^{\prime}, L^{\prime}\right) \rightarrow(K, L)$ we have $C_{w}\left(\phi^{f} z\right)=\phi^{\sharp} C_{w}(z)$.

We shall be interested in such cocycle formulas only in spaces (i.e. in singular complexes) but it is technically useful to have the definition for s.s. complexes in general. We note one consequence of this definition which we shall need below:

(4.2) If the cocycles $z$ and $z^{\prime}$ agree on all $n$-faces of a simplex $s \in K$, then $C_{w}(z)$ and $C_{w}\left(z^{\prime}\right)$ agree on $s$.

This is immediate from (b) of the definition above, taking $s$ together with its faces (and degeneracies) as a subcomplex $K^{\prime} \subset K$ and taking $\phi$ as the injection.

Finally, we shall use the following convention throughout Part II. Given an s.s. pair $(K, L)$ and a cochain $c \in C^{r}(L ; G)$, we shall identify $c$ also as a cochain of $K$ by defining it to be 0 on $K-L$. When we form the coboundary of such a cochain then we shall always use a subscript, e.g. $\delta_{L}$ or $\delta_{K}$, to indicate the complex (or space) in which the coboundary is being taken. We shall omit the subscript on $\delta$ only if no ambiguity is possible.

5. Cocycle expression for the second level extension invariant. We adopt here in detail all of the notations, definitions, etc. of $[8, \S 6]$, except that throughout, even for $n=1$, we shall consider only the case where $\Pi$ is abelian and does not operate on $G$. Let $C_{w}$ be a cocycle formula for the element $w^{a+1}$ of $H^{q+1}(\Pi, n ; G)$.

Now suppose that the cocycle $z$ in $Z^{n}(Y ; I I)$ represents $y^{n}$ and, since 
$w^{q+1} \vdash y^{n}=0$, that $C_{w}(z)=\delta v$ for some $v \in C^{q}(Y ; G)$. Suppose $z^{\prime}$ in $Z^{n}(X ; \Pi)$ represents $x^{n}$ and satisfies $j^{\dagger} z^{\prime}=f^{\ddagger} z$, in accordance with $[8,(6.1 \mathrm{c})]$. Then the desired expression for the second level extension invariant $[8,(6.6)]$ is given by

THEOREM 5.1. $C_{w}\left(z^{\prime}\right)-\delta_{X} f^{t} v$ is a cocycle in $Z^{q+1}(X, A ; G)$. These cocycles, for all possible choices of $z^{\prime}$ (with $z$ and $v$ fixed), represent precisely the elements of $\Lambda_{f}\left(y^{n}, w^{q+1}, x^{n}\right)$. Furthermore, if $z_{0}^{\prime}$ and $z_{1}^{\prime}$ are two such choices of $z^{\prime}$ and $z_{0}^{\prime}-z_{1}^{\prime}$ is cohomologous to 0 in $Z^{n}(X, A ; \Pi)$, then they yield the same element of $\Lambda_{f}\left(y^{n}, w^{q+1}, x^{n}\right)$ by the above formula.

REMARK 5.2. It follows that if $z_{0}^{\prime}$ is one such choice of $z^{\prime}$, we need consider only cocycles of the form $z_{0}^{\prime}+\delta_{X} z^{\prime \prime}$ for $z^{\prime \prime} \in Z^{n-1}(A ; \Pi)$.

Proof of theorem. To see that the cocycle $C_{w}\left(z^{\prime}\right)-\delta_{X} f^{\sharp} v$ vanishes on $A$ we note that, by naturality, $j^{\sharp} C_{w}\left(z^{\prime}\right)=f^{\sharp} C_{w}(z)=\delta_{A} f^{\prime} v$.

Now, as in $[8, \S 6]$, we consider the injections $j: A \rightarrow X$ and $j_{2}: A \rightarrow A_{f}$, and the deformation-retraction $\rho: A_{f} \rightarrow Y$; also let $i_{1}: X \rightarrow X \cup A_{f}$ and $i_{2}: A_{f} \rightarrow X \cup A_{f}$ be injections; clearly $f=\rho \circ j_{2}$.

We assert that there is a cocycle $u$ in $Z^{n}\left(X \cup A_{f} ; \Pi\right)$ such that $i_{1}^{*} u=z^{\prime}$ and $i_{2}^{\prime} u=\rho^{f} z$. To see this, we first define a cocycle $u^{\prime}$ in $S(X) \cup S\left(A_{f}\right)$ by setting $u^{\prime}=z^{\prime}$ on $S(X)$ and $u^{\prime}=\rho^{\ddagger} z$ on $S\left(A_{f}\right)$; this is meaningful since $z^{\prime}$ and $\rho^{\dagger} z$ agree on $S(A)$. Since we know from $[8,(19.1)]$ that $H^{n}\left(X \cup A_{f}, x_{0} ; \Pi\right)$ $\rightarrow H^{n}\left(S(X) \cup S\left(A_{f}\right), x_{0} ; \Pi\right)$ is onto, it follows that $u^{\prime}$ can be extended to a cocycle $u$ in $Z^{n}\left(X \cup A_{f} ; \Pi\right)$, which has the asserted properties.

This $u$ then represents an element $h^{n} \in H^{n}\left(X \cup A_{f}, x_{0} ; \Pi\right)$ and can be used to compute a unique element of $\Lambda_{f}\left(y^{n}, w^{q+1}, x^{n}\right)$ in accordance with the definition $[8,(6.6)]$. That is, we form $C_{w}(u)$ in $Z^{q+1}\left(X \cup A_{f} ; G\right)$ and then, since

$$
i_{2}^{\dagger} C_{w}(u)=C_{w}\left(i_{2}^{\ddagger} u\right)=C_{w}\left(\rho^{\ddagger} z\right)=\rho^{\dagger} \delta v=\delta_{\Delta_{f}}\left(\rho^{\ddagger} v\right) \text {, }
$$

we take the cocycle

$$
C_{w}(u)-\delta_{X} \cup_{A_{j}}\left(\rho^{t} v\right)
$$

which lies in $Z^{q+1}\left(X \cup A_{f}, A_{f} ; G\right)$ and is cohomologous to $C_{w}(u)$ in $X \cup A_{f}$. According to $\left[8,(6.6)\right.$ and (6.4)] the desired element of $\Lambda_{f}\left(y^{n}, w^{q+1}, x^{n}\right)$ is then represented (using the injection $\left.l_{1}:(X, A) \rightarrow\left(X \cup A_{f}, A_{f}\right)\right)$ by

$$
l_{1}^{t}\left(C_{w}(u)-\delta_{X} \cup_{A f}\left(\rho^{t} v\right)\right)=C_{w}\left(i_{1}^{*} u\right)-\delta_{X} j_{2}^{t} \rho^{t} v=C_{w}\left(z^{\prime}\right)-\delta_{X} f^{t} v .
$$

We have shown then that $C_{w}\left(z^{\prime}\right)-\delta_{X} f^{\prime} v$ represents an element of $\Lambda_{f}\left(y^{n}, w^{q+1}, x^{n}\right)$.

Conversely, if we are given an element of $\Lambda_{f}\left(y^{n}, w^{q+1}, x^{n}\right)$, then it must arise from some $h^{n} \in H^{n}\left(X \cup A_{f}, x_{0} ; \Pi\right)$ in the manner given by [8, (6.6)]. This $h^{n}$ will be represented by a cocycle $\bar{u} \in Z^{n}\left(X \cup A_{f} ; \Pi\right)$ such that $\left\{i_{1}^{t} \bar{u}\right\}$ $=x^{n}$ in $X$ and $\left\{i_{2}^{t} \bar{u}\right\}=\rho^{*} y^{n}$ in $A_{f}$. It follows that, for some $a \in C^{n-1}\left(A_{f}, x_{0} ; \Pi\right)$, $i_{2}^{*} \bar{u}=\rho^{f} z+\delta_{\Delta f} a$. We define $u \in Z^{n}\left(X \cup A_{f} ;\right.$ II $)$ by $u=\bar{u}-\delta_{X \cup \Lambda_{f}} a$.

Then $\{u\}=h^{n}$ and $i_{2}^{\dagger} u=\rho^{\dagger} z$; if we set $z^{\prime}=i_{1}^{\dagger} u$ then $\left\{z^{\prime}\right\}=x^{n}$ and $j^{\prime} z^{\prime}=f^{\sharp} z$. 
It follows from the argument above that the given element is represented by $C_{w}\left(z^{\prime}\right)-\delta_{X} f^{\prime} v$.

Finally, to prove the last statement of the theorem, suppose we have $u_{1} \in Z^{n}\left(X \cup A_{f} ; \Pi\right)$ such that $i_{1}^{t} u_{1}=z_{1}^{\prime}$ and $i_{2}^{t} u_{1}=\rho^{\prime} z$. Then $i_{1}^{t} u_{1}=z_{0}^{\prime}+\delta_{X} c$ for some $c \in C^{n-1}(X, A ; \Pi)$. We define $u_{0}$ to be $u_{1}-\delta_{X \cup \Delta_{f}} c$. Then $\left\{u_{0}\right\}=\left\{u_{1}\right\}$ and so $u_{0}$ yields, by the procedure of (5.3), (5.4) above, the same element of $\Lambda_{f}\left(y^{n}, w^{q+1}, x^{n}\right)$ as $u_{1}$. Furthermore $i_{1}^{t} u_{0}=z_{0}^{\prime}$ and $i_{2}^{t} u_{0}=i_{2}^{t} u_{1}=\rho^{t} z$ since $c$ vanishes on $A$. It follows then from (5.4) above that $C_{w}\left(z_{0}^{\prime}\right)-\delta_{X} f^{\prime} v$ and $C_{w}\left(z_{1}^{\prime}\right)$ $-\delta_{X} f^{t} v$ are cohomologous mod $A$. This completes the proof.

6. Cocycle expression for the homotopy invariant. We associate with the cocycle formula $C_{w}$ a function $\bar{C}_{w}$ which assigns to a pair of elements $b^{n} \in Z^{n}(X ; \Pi)$ and $c^{n-1} \in C^{n-1}(X, A ; \Pi)$ an element $\bar{C}_{w}\left(b^{n}, c^{n-1}\right)$ in $C^{q}(X, A ; G)$; $A$ may be vacuous here.

To define $\bar{C}_{w}$, let $d^{n} \in Z^{n}(X \times I ; \Pi)$ be the cocycle

$$
d^{n}=\rho_{0}^{t} b^{n}+\delta_{X \times I} c^{n-1}
$$

where $\rho_{0}$ is the projection $X \times I \rightarrow X$ (identifying $X$ with $X \times 0$ ). Let $u_{q}: s_{q} \rightarrow X$ be any singular $q$-simplex of $X$ and $U: s_{q} \times I \rightarrow X \times I$ the corresponding singular prism, where $U(a, t)=\left(u_{q}(a), t\right)$. Denoting by $v_{0}, \cdots, v_{q}, \bar{v}_{0}, \cdots, \bar{v}_{q}$ the vertices (in order) of $s_{q} \times I$ (where the $v_{i}$ are the vertices of $s_{q}$ and $\bar{v}_{i}=v_{i} \times 1$ ), and by $U_{f}$ the map of singular chains induced by $U$, we may consider the integral singular $(q+1)$-chain

$$
\gamma_{q+1}=U_{+} \sum_{i=0}^{q}(-1)^{i}\left(v_{0} v_{1} \cdots v_{i} \bar{v}_{i} \cdots \bar{v}_{q}\right)
$$

in $X \times I$. Then we define $\bar{C}_{w}\left(b^{n}, c^{n-1}\right)$ by

$$
\left(\bar{C}_{w}\left(b^{n}, c^{n-1}\right)\right)\left(u_{q}\right)=-\left(C_{w}\left(d^{n}\right)\right)\left(\gamma_{q+1}\right) .
$$

To see that (6.3) is 0 for $u_{q}$ in $A$, we observe that in this case it becomes (except for sign)

$$
\left(C_{w}\left(\rho_{0}^{\dagger} b^{n}\right)\right)\left(\gamma_{q+1}\right)=\left(\rho_{0}^{\dagger} C_{w}\left(b^{n}\right)\right)\left(\gamma_{q+1}\right)=\left(C_{w}\left(b^{n}\right)\right)\left(\rho_{0+} \gamma_{q+1}\right)
$$

and vanishes because $\rho_{0 f} \gamma_{a+1}$ is a degenerate chain.

We turn now to the homotopy problem and adopt the notations and definitions of [8, Part III, and, in particular, §10], except that, as in $\$ 5$ just above, we consider only the case where $\Pi$ is abelian and does not operate on $G$. Suppose, as in $\S 5$, that the cocycle $z \in Z^{n}(Y ; \Pi)$ represents $y^{n}$ and that $C_{w}(z)=\delta v$ for $v \in C^{q}(Y ; G)$. Also, in view of $[8,(10.2)$ and (9.8)], there is some $c \in C^{n-1}(X, A ; \Pi)$ such that

$$
\delta c=f+z-g \neq z .
$$

Theorem 6.5. (a) For Case I (homotopy rel. $A): \bar{C}_{w}\left(g^{t} z, c\right)-\left(f^{t} v-g^{t} v\right)$ is 
a cocycle in $Z^{q}(X, A ; G)$. These cocycles, for all $c$ satisfying (6.4) (with $z$ and $v$ fixed $)$, represent precisely the elements of $\Lambda_{f, o}\left(y^{n}, w^{q+1}\right)$.

(b) For Case II (where $A=x_{0}$ and the homotopy moves $x_{0}$ along $\omega \in \pi_{1}\left(Y, y_{0}\right)$ ) with $n>1: \Lambda_{f, q ; \omega}\left(y^{n}, w^{q+1}\right)$ is given by the same formula as in (a).

(c) For Case II, $n=1$. Let $\alpha=y^{1}(\omega) \in \Pi$ (interpreting $y^{1} \in \operatorname{Hom}\left(\pi_{1}\left(Y, y_{0}\right), \Pi\right)$ ) and let $\alpha$ also denote the constant cocycle with value $\alpha$ in $C^{0}(X, \Pi)$. Then the cocycles $\bar{C}_{w}\left(g^{\sharp} z, c-\alpha\right)-\left(f^{\sharp} v-g^{\sharp} v\right)$, for all $c$ satisfying (6.4), represent the elements of $\Lambda_{f, q ; \omega}\left(y^{1}, w^{q+1}\right)$.

Proof. Letting $\hat{X}=X \times 0 \cup A \times I \cup X \times 1$ (with $X$ identified with $X \times 0$ ), we define $F:\left(\hat{X}, x_{0}\right) \rightarrow\left(Y, y_{0}\right)$ in the usual way for both Case I and Case II; see $[8, \S 9]$. We consider Case I first. With $z, v$ and $c$ as above, we set

$$
\bar{z}=\rho_{0}^{\star} g^{\star} z+\delta_{X \times I} c .
$$

Then, if $\bar{j}$ is the injection $\hat{X} \rightarrow \bar{X}=X \times I, \bar{j}^{+} \bar{z}$ agrees with $F^{\sharp} z$ on all singular simplexes in $S^{\prime}=S(X \times 0) \cup S(A \times I \cup X \times 1)$. Since $H^{n}\left(S(\hat{X}), S^{\prime} ; \Pi\right)$ is clearly 0 , it follows that there is a cochain $d \in C^{n-1}\left(S(\hat{X}), S^{\prime} ; \Pi\right)$ such that $\bar{j}^{\ddagger} \bar{z}-F^{\sharp} z$ $=\delta d$. We set

$$
z^{\prime}=\bar{z}-\delta_{X \times I} d \quad \text { in } \quad Z^{n}(\bar{X} ; \Pi)
$$

so that now $\bar{j}^{\sharp} z^{\prime}=F^{\sharp} z$.

According to Theorem 5.1 above and the definition of $\Lambda_{f, g}$ in $[8,(10.5)]$, if we now form the cocycle

$$
C_{w}\left(z^{\prime}\right)-\delta_{X \times I} F \neq v \text { in } Z^{q+1}(\bar{X}, \hat{X} ; G),
$$

representing an element of $H^{q+1}(\bar{X}, \hat{X} ; G)$, and then carry this element back to $H^{q}(X, A ; G)$ by the isomorphism $\Delta^{-1}$ of $[8,(9.1)]$, we get an element of $\Lambda_{f, o}\left(y^{n}, w^{q+1}\right)$. A cocycle formula for $\Delta^{-1}$ is readily given. Namely, for a cocycle $\bar{c}$ in $Z^{q+1}(\bar{X}, \hat{X} ; G)$, we form $\tau(\bar{c})$ in $Z^{q}(X, A ; G)$ by setting

$$
(\tau(\bar{c}))\left(u_{q}\right)=-\bar{c}\left(\gamma_{q+1}\right)
$$

where $u_{q}$ and $\gamma_{q+1}$ are as in (6.3); then $\Delta^{-1}\{\bar{c}\}=\{\tau(\bar{c})\}$. This is well known; a proof (for the simplicial case, but easily modified) is given in $[6$, p. 564].

If we apply (6.9) to (6.8), the first term becomes $-\left(C_{w}\left(z^{\prime}\right)\right)\left(\gamma_{q+1}\right)$. But it is clear that $\delta_{X \times I} d$ vanishes on every $n$-face of every simplex in $\gamma_{q+1}$; hence, by (4.2) above, $\left(C_{w}\left(z^{\prime}\right)\right)\left(\gamma_{q+1}\right)=\left(C_{w}(\bar{z})\right)\left(\gamma_{q+1}\right)$. Thus, by (6.3), this first term is just $\left(\bar{C}_{w}\left(g^{\sharp} z, c\right)\right)\left(u_{q}\right)$.

The second term becomes $\left(F^{\prime} v\right)\left(\partial \gamma_{q+1}\right)$. Now for any $u_{q-1}$ and corresponding $\gamma_{q},\left(F^{*} v\right)\left(\gamma_{q}\right)=0$; this is so by the definition of $F^{*} v$ as a cochain of $\bar{X}$ if $u_{q-1}$ is not in $A$; and if $u_{q-1}$ is in $A$, then $\left(F^{*} v\right)\left(\gamma_{q}\right)=\left(\rho_{0}^{*} f^{\#} v\right)\left(\gamma_{q}\right)=\left(f^{*} v\right)\left(\rho_{0} \gamma_{q}\right)$ $=0$ because $\rho_{0} \gamma_{q}$ is degenerate. It follows then from the usual formula for $\partial \gamma_{q+1}$ (see [4, p. 195]) that $\left(F^{*} v\right)\left(\partial \gamma_{q+1}\right)$ is $\left(g^{*} v\right)\left(u_{q}\right)-\left(f^{\#} v\right)\left(u_{q}\right)$.

We conclude then that the formula given in (a) of the theorem represents, 
for each $c$, an element of $\Lambda_{f, o}\left(y^{n}, w^{q+1}\right)$.

To prove the converse, suppose $h \in \Lambda_{f, 0}\left(y^{n}, w^{q+1}\right)$. We know from Theorem 5.1 and $[8,(10.5)]$ that there is a cocycle $z_{1}^{\prime}$ in $Z^{n}(\bar{X} ; \Pi)$, with $\bar{j}^{\ddagger} z_{1}^{\prime}=F^{\ddagger} z$, such that

$$
h=\Delta^{-1}\left\{C_{w}\left(z_{1}^{\prime}\right)-\delta_{X \times I} F+v\right\}
$$

where $\Delta^{-1}$ is as above.

Now the cocycle $z_{1}^{\prime}-\rho_{0}^{\prime} g^{\dagger} z$ clearly vanishes on $A \times I \cup X \times 1$ and it follows that there is a cochain $e$ in $C^{n-1}(\bar{X}, A \times I \cup X \times 1 ; \Pi)$ such that $\delta e=z_{1}^{\prime}-\rho_{0}^{\dagger} g^{\dagger} z$. We decompose $e$ by writing it as $e=c-d+e^{\prime}$ where $c \in C^{n-1}(X, A ; \Pi)$, $d \in C^{n-1}\left(S(\hat{X}), S^{\prime} ; \Pi\right)$ and $e^{\prime} \in C^{n-1}(\bar{X}, \hat{X} ; \Pi), S^{\prime}$ being as defined above following (6.6). Note that $\delta_{X} c=f^{\sharp} z-g^{\ddagger} z$. If we now set

$$
z^{\prime}=\rho_{0}^{t} g^{\prime} z+\delta_{X \times I} c-\delta_{X \times I} d
$$

then $z_{1}^{\prime}-z^{\prime}=\delta e^{\prime}$; also $j^{\prime} z^{\prime}=\bar{j}^{\sharp} z_{1}^{\prime}=F^{\prime} z$. Hence, by Theorem 5.1, we get the same element $h$ if we use $z^{\prime}$ instead of $z_{1}^{\prime}$ in (6.10). It follows then from the first part of the proof that this $h$ is represented by $\bar{C}_{w}\left(g^{\ddagger} z, c\right)-\left(f^{t} v-g^{\ddagger} v\right)$. This completes the proof of (a).

The proof for Case II is essentially the same. For $n>1$ we define $\bar{z}$ by (6.6) again; for $n=1$ we use the same definition with $c$ replaced by $c-\alpha$. We denote by $S^{\prime \prime} \subset S(\bar{X})$ the singular subcomplex given by $S(X \times 0) \cup S(X \times 1)$ together with the singular 1-simplex $x_{0} \times I$. For the present case it is clear then that $\bar{j}^{\ddagger} \bar{z}$ agrees with $F_{z}^{*}$ on $S^{\prime \prime}$ and hence (since $H^{*}\left(S(\hat{X}), S^{\prime \prime} ; \Pi\right)=0$ ) there is a $d$ in $C^{n-1}\left(S(\hat{X}), S^{\prime \prime} ; \Pi\right)$ such that $\bar{j}^{\ddagger} \bar{z}-F^{*} z=\delta d$. The remainder of the argument then goes as above.

7. Product and Bockstein operations. We look first at the expression $\bar{C}_{w}$ of $\S 6$ for the case where $w \in H^{r n}(\Pi, n ; G)$ is the $r$-fold product operation. That is, given $z^{\prime} \in Z^{n}(X ; \Pi)$ we suppose $w \vdash\left\{z^{\prime}\right\}=\left\{\left(z^{\prime}\right)^{r}\right\}$ in $H^{r n}(X ; G)$, the $r$-fold product being taken relative to some given $r$-linear map $\Pi \times \Pi \times \ldots$ $\times \Pi \rightarrow G$.

Theorem 7.1. Let $b^{n}$ and $c^{n-1}$ be as in $\$ 6$ and define $a^{n} \in C^{n}(X ; \Pi)$ by $a^{n}=b^{n}+\delta_{X} c^{n-1}$. Then, for the $w$ just described,

$$
\bar{C}_{w}\left(b^{n}, c^{n-1}\right)=\sum_{k=0}^{r-1}(-1)^{k n}\left(a^{n}\right)^{k} \cdot c^{n-1} \cdot\left(b^{n}\right)^{r-1-k} .
$$

Proof. Returning to(6.3) and (6.2), we look at $\left(d^{n}\right)^{r}\left(U_{t}\left(v_{0} v_{1} \cdots v_{i} \bar{v}_{i} \cdots \bar{v}_{q}\right)\right)$ where now $q=r n-1$. It is clear from (6.1) that this is 0 except for $i=(k+1) n-1$ where $k=0,1,2, \cdots, r-1$. For such an $i$ it becomes

$$
\begin{aligned}
\left(a^{n} u_{q f}\left(v_{0} \cdots v_{n}\right)\right) \cdots\left(a^{n} u_{q f}\left(v_{(k-1) n} \cdots v_{k n}\right)\right) \\
\cdot\left((-1)^{n} c^{n-1} u_{q f}\left(v_{k n} \cdots v_{(k+1) n-1}\right)\right) \\
\cdot\left(b^{n} u_{q f}\left(v_{(k+1) n-1} \cdots v_{(k+2) n-1}\right)\right) \cdots\left(b^{n} u_{q f}\left(v_{(r-1) n-1} \cdots v_{r n-1}\right)\right)
\end{aligned}
$$


or $\left((-1)^{n}\left(a^{n}\right)^{k} \cdot c^{n-1} \cdot\left(b^{n}\right)^{r-1-k}\right)\left(u_{q}\right)$. This, with (6.2) and (6.3), proves the theorem.

We look next at another case which occurs in practice, namely where $w \in H^{q+1}\left(Z_{l}, n ; Z\right)$ is the Bockstein operation $\beta \in H^{n+1}\left(Z_{l}, n ; Z\right)$ followed by the $r$-fold product. Thus $q+1=r(n+1)$. We can give a cocycle formula for $w$ as follows.

Let $\eta: Z_{\imath} \rightarrow Z$ and $C^{*}\left(X ; Z_{\imath}\right) \rightarrow C^{*}(X ; Z)$ be as defined in $\S 3$ above. Then, given $z^{\prime} \in Z^{n}\left(X ; Z_{l}\right)$, the cocycle

$$
C_{w}\left(z^{\prime}\right)=\left(\frac{1}{l} \delta \eta z^{\prime}\right)^{r}
$$

represents $w \vdash\left\{z^{\prime}\right\}$ in $H^{q+1}(X ; Z)$.

THEOREM 7.4. Let $b^{n}$ and $c^{n-1}$ be as in $\S 6$ and define $a^{n} \in C^{n}(X ; \Pi)$ by $a^{n}=b^{n}+\delta_{X} c^{n-1}$. Then, for the w just described,

$\bar{C}_{w}\left(b^{n}, c^{n-1}\right)$

$$
=\sum_{k=0}^{r-1}(-1)^{k(n+1)}\left(\frac{1}{l} \delta \eta a^{n}\right)^{k} \cdot \frac{1}{l}\left(\eta a^{n}-\eta b^{n}-\delta \eta c^{n-1}\right) \cdot\left(\frac{1}{l} \delta \eta b^{n}\right)^{r-1-k}
$$

Proof. Starting from (6.2), (6.3) and (7.3) we get easily (as with (7.2) above) that $(l)^{r}\left(\bar{C}_{w}\left(b^{n}, c^{n-1}\right)\right)\left(u_{q}\right)$ is

$$
\begin{aligned}
&-\sum_{k=0}^{r-1}(-1)^{j}\left(\left(\delta \eta a^{n}\right)^{k} u_{q f}\left(v_{0} \cdots v_{k(n+1)}\right)\right) \\
& \cdot Q \cdot\left(\left(\delta \eta b^{n}\right)^{r-1-k} u_{q f}\left(v_{k(n+1)+n} \cdots v_{q}\right)\right)
\end{aligned}
$$

where

$$
Q=\sum_{m=j}^{j+n}(-1)^{m-j}\left(\delta \eta d^{n}\right) U_{t}\left(v_{j} \cdots v_{m} \bar{v}_{m} \cdots \bar{v}_{j+n}\right)
$$

and $j=k(n+1)$. By straightforward computation, using (6.1), we get

$$
\begin{aligned}
Q= & \left(\eta d^{n}\right) U_{t}\left(\bar{v}_{j} \cdots \bar{v}_{j+n}\right) \\
& +(-1)^{n-1}(-1)^{n+1}\left(\eta d^{n}\right) U_{t}\left(v_{j} \cdots v_{j+n-1} \bar{v}_{j+n-1}\right) \\
& +(-1)^{n} \sum_{i=j}^{j+n-1}(-1)^{i-j}\left(\eta d^{n}\right) U_{t}\left(v_{j} \cdots \hat{v}_{i} \cdots v_{j+n} \bar{v}_{j+n}\right) \\
& +(-1)^{n}(-1)^{n+1}\left(\eta d^{n}\right) U_{t}\left(v_{j} \cdots v_{j+n}\right)
\end{aligned}
$$

since $\left(\eta d^{n}\right) U_{f}\left(v_{j} \cdots \theta_{i} \cdots v_{m} \bar{v}_{m} \cdots \bar{v}_{j+n}\right)=0$ except when $m=j+n$, and the corresponding expression with $\bar{v}_{i}$ omitted instead of $v_{i}$ is 0 except when $m=j+n-1$. The first and last terms of (7.7) combine to give

$$
-\left(\eta a^{n}-\eta b^{n}\right) u_{q f}\left(v_{j} \cdots v_{j+n}\right) \text {. }
$$


For the remaining terms we observe first that

$$
\begin{aligned}
& \left(\eta \delta c^{n-1}\right) U_{t}\left(v_{j} \cdots \hat{v}_{i} \cdots v_{j+n} \bar{v}_{j+n}\right) \\
& \quad=\left\{\begin{array}{lr}
\left(\eta c^{n-1}\right) U_{t}\left(v_{j} \cdots \hat{v}_{i} \cdots v_{j+n}\right) & \text { if } n \text { even, } \\
l-\left(\eta c^{n-1}\right) U_{t}\left(v_{j} \cdots \hat{v}_{i} \cdots v_{j+n}\right) & \text { if } n \text { odd }
\end{array}\right.
\end{aligned}
$$

and similarly for the second line of (7.7). The middle two lines of (7.7) give then

$$
(-1)^{n} \sum_{i=j}^{j+n}(-1)^{i-j}\left(l-\left(\eta c^{n-1}\right) U_{f}\left(v_{j} \cdots \hat{\delta}_{i} \cdots v_{j+n}\right)\right)
$$

for $n$ odd and the same expression with $\eta c^{n-1}$ instead of $l-\eta c^{n-1}$ for $n$ even. In either case, this becomes

$$
\left(\delta \eta c^{n-1}\right) u_{q f}\left(v_{j} \cdots v_{j+n}\right) .
$$

Inserting (7.8) and (7.11) in (7.7), and the result in (7.5), then completes the proof.

8. Proofs for the material of $\S 1$. We begin with Theorem 1.1 and with (1.3a) for this case. By [8, Corollary 10.10 and Remark 10.11] we have $f \cong g$ rel. $A$ if and only if $(f-g)^{*} y^{1}=0,(f-g)^{*} y^{q}=0$ and $0 \in \Lambda_{f, o}\left(y^{1}, w^{q+1}\right)$ for the nonzero element $w^{q+1}$ of $H^{q+1}\left(Z_{2}, 1 ; Z\right)$; here $Z_{2}$ operates trivially for $q$ odd, nontrivially for $q$ even.

The invariant $\Lambda_{f, 0}\left(y^{1}, w^{q+1}\right)$ lies in $H^{q}\left(M, A ; Z^{f}\right)$, where $Z^{f}$ is as in (1.3b), and consists of a single element which is either 0 or of order 2 ; see $[8,(10.5)$, (14.1) and (14.3)]. Clearly $H^{q}\left(M, A ; Z^{f}\right)$ is the direct sum of a free group and a group all of whose elements have order at most 2 , and this latter group maps monomorphically under the coefficient homomorphism $Z^{f} \rightarrow Z_{2}$. Hence, by $[8,(14.15)], 0=\Lambda_{f, o}\left(y^{1}, w^{q+1}\right)$ if and only if $0=\Lambda_{f, o}\left(y^{1}, w^{q+1}\right) \in H^{q}\left(M, A ; Z_{2}\right)$, where $w^{q+1}$ is the nonzero element of $H^{q+1}\left(Z_{2}, 1 ; Z_{2}\right)$.

Since this $w^{q+1}$ is just the $(q+1)$-fold product $[9$, p. 204], we may compute $\Lambda_{f, g}\left(y^{1}, w^{q+1}\right)$ by Theorems 6.5 and 7.1 above. This proves then the continuous case, (1.3a) above, of Theorem 1.1. If $f$ and $g$ are simplicial maps, we may restrict our cocycle representative of $\Lambda_{f, g}\left(y^{1}, w^{q+1}\right)$ to the simplicial complex and compute it there; since $v$ is a cocycle when restricted to the simplicial complex, $f^{t} v-g^{t} v$ must, by (ii), be a coboundary on the simplicial complex and therefore may be omitted from the expression in (iii). This completes the proof of Theorem 1.1.

The proof of Theorem 1.2, and of (1.3a) for this case, is exactly the same, using now part (c) of Theorem 6.5 and the fact that

$$
\sum_{k=0}^{q}(f \sharp z)^{k} \cdot 1 \cdot(g+z)^{q-k}
$$


represents $(q+1) f^{*}\left(y^{1}\right)^{q}$ in $H^{q}\left(M, x_{0} ; Z_{2}\right)$, where 1 in (8.1) is the generator of $Z^{0}\left(M ; Z_{2}\right)$. The use of $f^{*} y^{q}=(-1)^{q+1} g^{*} y^{q}$ in (ii) is justified by $[8,(9.10)$ and (18.5)].

Proof for (1.3b). For the case where $A=x_{0}$ this is given in [7, Theorems IIa and IIIc]. The proof (contained in $[7, \$ \$ 11$ and 12]) generalizes without essential change to arbitrary $A$ and is left to the reader.

Proof for (1.3c). If $f$ and $g$ are orientation-true, $H^{q}\left(M, A ; Z^{\prime}\right)$ is freeabelian. Consequently $\Lambda_{f, o}\left(y^{1}, w^{\prime q+1}\right)$, being of order at most 2 (see above), is necessarily 0.

Proof for (1.3d). If $f$ and $g$ are not orientation-true, $H^{q}\left(M, x_{0} ; Z^{f}\right)$ is cyclic of order 2 . Hence the first paragraph of (1.3d) follows from (1.3b). The assertion of the second paragraph with regard to relative homotopy follows at once from $[8,(10.12)]$, and the statement about free homotopy is immediate from a comparison of (iii) of Theorem 1.1 with (iii) of Theorem 1.2.

\section{Proofs for the material of $\$ \$ 2$ and 3 .}

Proof for (2.2a) and Theorem 2.1. The proof is the same as that for (1.3a) and Theorem 1.1, except that here the situation is simpler because $H^{2 q+1}(\mathfrak{B} ; Z)$ $=0$ and the generator $w^{2 q+2}$ in $H^{2 q+2}(Z, 2 ; Z)$ is already, as a cohomology operation, just the $(q+1)$-fold product with integer coefficients $[9,(29.4)]$. The proof then follows from $[8,(10.10)$ and (10.11)] and Theorems 6.5 and 7.1 above.

Proof for (2.2b). The enumeration follows from $[8,(10.12)]$ and the expression for $\Lambda_{f, f}\left(y^{2}, w^{2 q+2}\right)$ given by (ii) of Theorem 2.1 .

Proof for (2.2c). This is immediate here since $\left\{f^{\prime} z\right\}=\left\{g^{\prime} z\right\}$ and $c$ can be varied only by a cocycle in $Z^{1}(X, A ; Z)$. It is in any case given by the general property $[8,(15.9)]$.

Proof for (3.2a) and Theorem 3.1. It is well known that a generator of $H^{2 q+2}\left(Z_{l}, 1 ; Z\right)$, as a cohomology operation, is just the Bockstein operator $\beta \in H^{2}\left(Z_{l}, 1 ; Z\right)$ followed by the $(q+1)$-fold product with integer coefficients; see, for example, $[3$, p. 94]. Then (3.2a) and Theorem 3.1 follow at once from $[8,(10.10)$ and $(10.11)]$ and Theorems 6.5 and 7.4 above. The final statement, concerning homotopy rel. $\omega$, is immediate from applying Theorem 7.4 to Theorem 6.5c and comparing with (iii) of the present theorem; (it is in any case obvious that the identity map of $L$ is homotopic to itself rel. $\omega$ for every $\left.\omega \in \pi_{1}\left(L, y_{0}\right)\right)$.

Proof for (3.2b). For the case where $X$ is a $(2 q+1)$-manifold and $A=x_{0}$, this is given in $[7$, Theorems IIa and III]. The proof (contained in $[7, \S \S 11$, 12]) generalizes without essential change to the present case and is left to the reader.

Proof for (3.2c). This follows from $[8,(14.3)$ and (16.1)].

Proof for (3.2d). This is given by $[8,(10.12)]$. 


\section{APPENDIX}

Difference homomorphisms. Let $(X, A)$ be a topological pair with base point $x_{0} \in A \subset X$. We denote $\bar{X}=X \times I, I$ being the unit interval $0 \leqq t \leqq 1$, identify $X$ with $X \times 0$ and set $X_{1}=X \times 1$ and $\hat{X}=X \cup(A \times I) \cup X_{1}$. We let

$$
\Delta: H^{m}(X, A ; G) \approx H^{m+1}(\bar{X}, \hat{X} ; G)
$$

be the coboundary homomorphism of the triad $\left(\bar{X}, X, X_{1} \cup(A \times I)\right)$; it is clearly an isomorphism for any abelian group $G$.

We consider maps $f, g:\left(X, x_{0}\right) \rightarrow\left(Y, y_{0}\right)$ with $f|A=g| A$ and define

$$
F:\left(\hat{X}, x_{0}\right) \rightarrow\left(Y, y_{0}\right)
$$

by setting $F(x, 0)=f(x), F(x, 1)=g(x)$ and $F(x, t)=f(x)=g(x)$ for $x \in A$, all $t$.

Let $G$ be a simple coefficient group and consider the composition

$$
H^{m}(Y ; G) \stackrel{F^{*}}{\rightarrow} H^{m}(\hat{X} ; G) \stackrel{\delta}{\rightarrow} H^{m+1}(\bar{X}, \hat{X} ; G) .
$$

Then the difference homomorphism

$$
(f-g)^{*}: H^{m}(Y ; G) \rightarrow H^{m}(X, A ; G)
$$

is defined by setting $(f-g)^{*}=\Delta^{-1} \circ \delta \circ F^{*}$. It is not difficult to see that, if $z$ is a singular cocycle representing an element of $H^{m}(Y ; G)$, then $(f-g)^{*}\{z\}$ is represented by the cocycle $f^{\sharp} z-g^{\sharp} z$ in $Z^{m}(X, A ; G)$.

In $\S 1$, and again in (3.2e), reference is made to difference homomorphisms with local coefficients where the image space $Y=P$ is real projective $q$-space. Here we are using as our coefficients the twisted integers $Z^{t}$ in $P$, i.e., the integers with the generator of $\pi_{1}\left(P, y_{0}\right)$ operating nontrivially. The map $f:\left(X, x_{0}\right) \rightarrow\left(P, y_{0}\right)$ then induces local coefficients in $X$, namely the integers with $\gamma \in \pi_{1}\left(X, x_{0}\right)$ operating trivially if $f_{*}(\gamma)=0$, nontrivially otherwise; we denote these local coefficients $Z^{f}$.

If we let $y^{1}$ be the generator of $H^{1}\left(P ; Z_{2}\right)$ then the condition $(f-g)^{*} y^{1}=0$ (which holds in all cases under consideration) is easily seen to imply that the local coefficient group $Z^{F}$ in $\hat{X}$, induced by the map $F$ of (9.2), can be extended in a unique way to a local coefficient group in $\bar{X}$ which we may call $Z^{F}$ again.

With these remarks we need only to observe that (9.1) and (9.3) are now valid with $Z^{t}$ in $Y=P$ and $Z^{F}$ in $\bar{X}$. Since it is clear that the restriction of $Z^{F}$ to $X \subset \bar{X}$ yields the local group $Z^{f}$ in $X$, the definition $(f-g)^{*}=\Delta^{-1} \circ \delta \circ F^{*}$ now gives us the difference homomorphism

$$
(f-g)^{*}: H^{m}\left(P ; Z^{t}\right) \rightarrow H^{m}\left(X, A ; Z^{f}\right) .
$$

It is this homomorphism (9.5) which is used in (ii) of Theorem 1.1 and again 
in (1.3)b, footnote 5 and (ii) of (3.2e). A cocycle formula can be given for $(f-g)^{*}$ here also but it is somewhat more cumbersome than for the case of simple coefficients above and we omit it.

\section{REFERENCES}

1. H. Cartan, Algèbres d'Eilenberg-MacLane et homotopie, Séminaire Henri Cartan 1954/ 1955, Secrétariat Mathématique, Paris, 1955.

2. B. Eckmann, Sur les applications d'un polyedre dans un espace projectif complexe, C. R. Acad. Sci. Paris 228 (1949), 1397-1399.

3. S. Eilenberg and S. MacLane, Homology of spaces with operators. II, Trans. Amer. Math. Soc. 65 (1949), 49-99.

4. S. Eilenberg and N. Steenrod, Foundations of algebraic topology, Princeton Univ. Press, Princeton, N. J., 1952.

5. I. I. Gordon, The classification of the mappings of a complex in a projective space, C. R. (Doklady) Acad. Sci. URSS 65 (1949), 441-444. (Russian)

6. P. Olum, On mappings into spaces in which certain homotopy groups vanish, Ann. of Math. 57 (1953), 561-574. 480.

7. - Mappings of manifolds and the notion of degree, Ann. of Math. 58 (1953), 458-

8. - Invariants for effective homotopy classification and extension of mappings, Mem. Amer. Math. Soc. No. 37, 1961.

9. J.-P. Serre, Cohomologie modulo 2 des complexes d'Eilenberg-MacLane, Comment. Math. Helv. 27 (1953), 198-232.

10. H. Wada, On mappings from complexes into the complex projective space, Tohoku Math. J. 4 (1952), 69-76.

11. - Über die Abbildungen vom Komplexe auf den ungeraden dimensionalen reellen projektiven Raum, Tôhoku Math. J. 4 (1952), 231-241.

12. —, Irrtümer, Tóhoku Math. J. 5 (1954), 313.

Cornell University, ITHACA, NEW YORK 